\title{
Lamellar Separation in the Human Lens: The Case for Fibre Folds. A Combined In Vivo and Electron Microscopy Study
}

\author{
NICHOLAS A. P. BROWN, ${ }^{*}$ GIJS VRENSEN, ${ }^{*}$ G. ADRIEN SHUN-SHIN, ${ }^{*}$ and \\ BEN WILLEKENS** \\ Oxford and Amsterdam
}

\begin{abstract}
Summary
Lamellar separation is seen as parallel lines in the lens cortex. It has been the subject of a joint study between Oxford and Amsterdam. The condition was studied in vivo by macro photography and in vitro by scanning electron micrscopy.

The lines are seen to run concentric with the lens equator, crossing the lens fibres at right angles. The lines are commonly limited by a spoke cataract, a water cleft, or a lens suture. The line may continue beyond these features with a change in direction. The lines may branch and rejoin. The lines extend in depth into the lens, polarised light showing that the lines are due to reflection.

The name 'lamellar separation' implied that the appearance is due to separation between the lens lamellae. Lamellar separation is now shown by electron microscopy to be due to folds crossing the lens fibres.

The clinical study showed the lines occurring with spoke cataract and the electron microscopy showed the association with the novel finding of peripheral breaks in the fibres. A new name 'Fibre Folds' is proposed.
\end{abstract}

There is a pathological change in the lens of the eye, which has been known since the slitlamp microscope was first used and this change has contrinued to be known by the name that it was first given, 'lamellar (or lamellary) separation'. The name was chosen, probably by Vogt, 'lamelläre Zerklüftung', ${ }^{1}$ in the first place, to describe the appearance of more or less parallel lines in the lens cortex, which were considered to represent separations between adjacent lamellae of lens fibres. Other early writers on slit-lamp microscopy of the eye, such as Berliner ${ }^{2}$ in 1949 followed
Vogt's terminology and accepted that the basis was that of separation, which Berliner compared to the splitting apart of the hull of a wooden boat. Until now there appears to have been no attempt to elucidate the true nature of lamellar separätion.

Lamellar separation is a common finding in the cortex of the senile lens and its prevalence has been estimated by Schild ${ }^{3}$ as $7 \%$ in the $50-60$ year age group, $18 \%$ in the $60-70$ year age group and $52 \%$ in the $70-80$ year age group. The same author observed bilaterality in $75 \%$ of cases. Lamellar separation can be

From: * The Nuffield Laboratory of Opthalmology, Walton Street, Oxford OX2 6AW.

** The Netherlands Ophthalmic Research Institute, PO Box 12141, 1100 AC Amsterdam, The Netherlands. Correspondence to: Mr. N A P Brown, The Nuffield Laboratory of Ophthalmology, Walton Street, Oxford OX2 6AW. 
observed in both the anterior and posterior cortex of the lens, but is less easily observed in the posterior cortex and considered to be less common at that site. ${ }^{3}$ The most common direction of the lines observed by Schild and by $\operatorname{Vogt}^{1}$ was from lower temporal to upper nasal.

\section{Materials and Methods}

The study was undertaken in two parts; in vivo and in vitro:

\section{In Vivo Study}

Patients with lamellar separation were identified from patients attending the Clinical Cataract Research Unit at Oxford. These patients were studied at the slit-lamp to note the site of lamellar separation in the anterior part of the cortex of the lens and the relationship of lamellar separation to the lens sutures, to cortical cataract and to water clefts.

Patients were photographed using the macro camera. ${ }^{4}$ Selected images were measured at the Documator (Zeiss Jena) and the results compensated for corneal magnification as previously described. ${ }^{5}$

\section{In Vitro Study}

Cadaver lenses were removed by cutting the zonules from eyes that had been used to provide corneal grafts. These lenses were preserved in castor oil and studied at the slit-lamp microscope at the Clinical Cataract Research
Unit, Oxford. In those lenses in which lamellar separation was seen (9 out of 22 lenses examined), the site of the lamellar separation was identified with respect to flecks of iris pigment epithelium on the capsule. The nine lenses with lamellar separation were sent to Amsterdam for scanning electron microscopy. One lens without lamellar separation was sent as a masked control.

\section{Electron Microscopy}

The selected in vitro lenses, preserved in castor oil for slit-lamp examination, were immersed in a phosphate buffered fixative containing $4 \%$ formaldehyde and $1 \%$ gluteraldehyde, $\mathrm{pH} 7.3$. After fixation for two days to several weeks, the lenses were thoroughly rinsed in a buffer solution, dissected, dehydrated and critical point dried with $\mathrm{CO}_{2}$. Subsequently the dried specimens were fractured at the site of interest as indicated in the slit-lamp drawings of the particular lens. The fractured pieces were glued on pin-type specimen mounts, gold coated and inspected in a Philips SEM 505 scanning electron microscope using a secondary emission detector. New fracture planes were created and gold coated, as previously described. ${ }^{6,7}$

\section{Results}

In Vivo Study (Table I)

Lamellar separation is observed in all cases to occur at a mid depth in the cortex of the lens

Table I Lamellar separation

Twenty patients: female 12, male 8; mean age 75.5; thirty lenses affected by lamellar separation

\begin{tabular}{|c|c|c|c|c|c|}
\hline \multicolumn{6}{|c|}{ Site of occurrence of lamellar separation in affected lenses } \\
\hline $\begin{array}{l}\text { Lower nasal } \\
\quad 85 \%\end{array}$ & \multicolumn{2}{|c|}{$\begin{array}{l}\text { Lower temporal } \\
65 \%\end{array}$} & $\begin{array}{l}\text { Upper nasal } \\
19 \%\end{array}$ & \multicolumn{2}{|c|}{$\begin{array}{l}\text { Upper temporal } \\
19 \%\end{array}$} \\
\hline \multicolumn{6}{|c|}{ Termination of lines } \\
\hline \multirow[t]{2}{*}{$\begin{array}{l}\text { Spoke } \\
35 \%\end{array}$} & \multicolumn{2}{|c|}{$\begin{array}{l}\text { Water cleft } \\
17 \%\end{array}$} & $\begin{array}{l}\text { Suture } \\
15 \%\end{array}$ & \multicolumn{2}{|c|}{$\begin{array}{l}\text { Indeterminate } \\
\qquad 34 \%\end{array}$} \\
\hline & \multicolumn{3}{|c|}{$\begin{array}{l}\text { Relationship to other lens pathology } \\
\text { Per cent occurrence in affected lenses }\end{array}$} & & \\
\hline $\begin{array}{l}\text { Thinned subcapsular } \\
\text { clear zone } \\
67 \%\end{array}$ & $\begin{array}{c}\text { Spoke } \\
\text { cataract } \\
96 \%\end{array}$ & $\begin{array}{l}\text { Water } \\
\text { clefts } \\
33 \%\end{array}$ & $\begin{array}{c}\text { Subcapsular } \\
\text { cataract } \\
11 \%\end{array}$ & $\begin{array}{c}\text { Nuclear } \\
\text { brunescence } \\
19 \%\end{array}$ & $\begin{array}{c}\text { Nuclear } \\
\text { scatter } \\
19 \%\end{array}$ \\
\hline
\end{tabular}

Subcapsular clear zone thinning $=$ Oxford Grade 2 or less.

Nuclear brunescence $=$ Oxford Grade 3 or more.

Nuclear scatter (White scatter) = Oxford Grade 3 or more. 


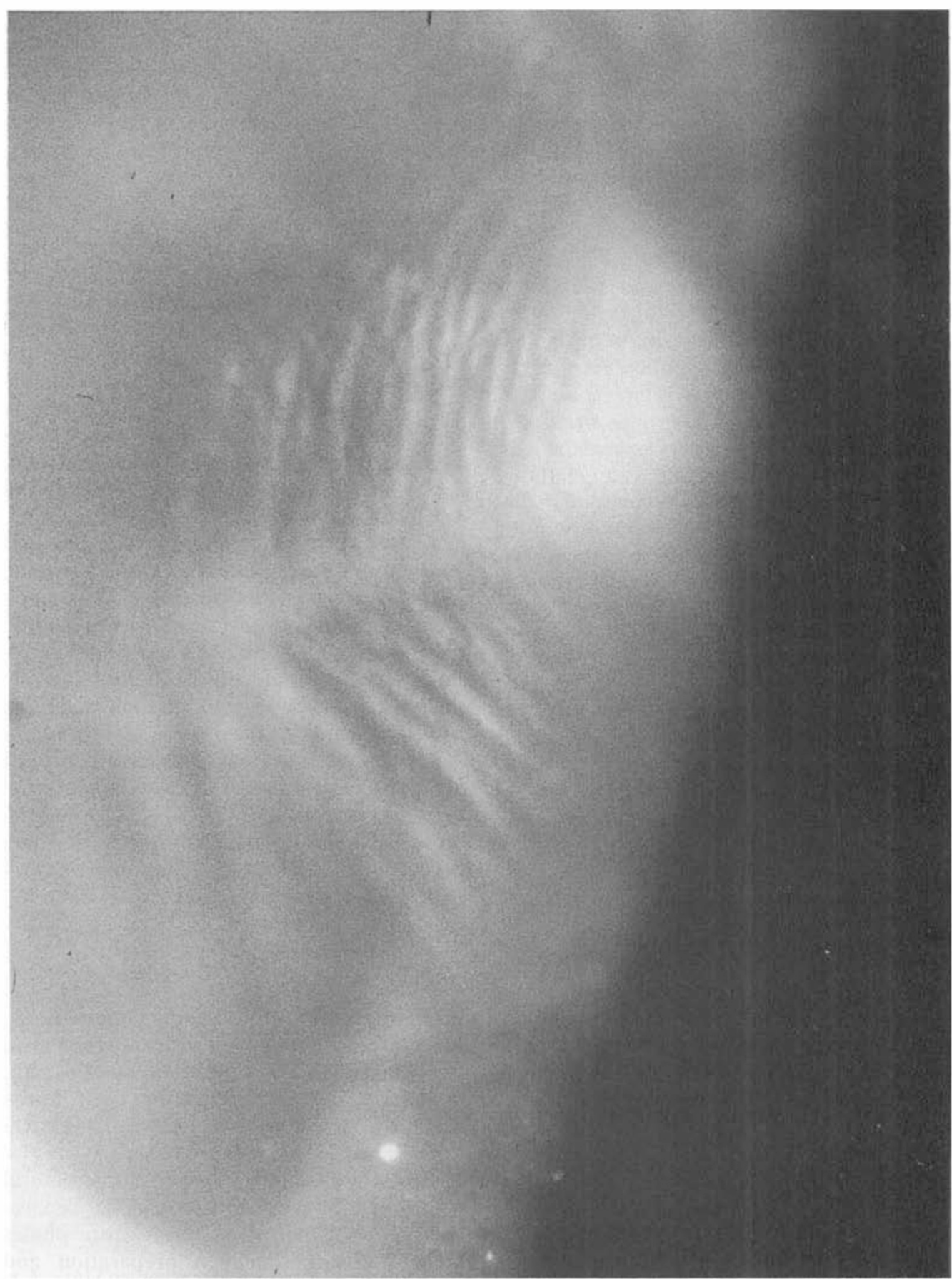

Fig. 1. Macrophotograph (primary magnification x10) of lamellar separation in the lens of the living eye by focal illumination. The fibre folds are seen to be interrupted by a lens suture making the 'herring bone' pattern. 
(cortical zone $\mathrm{C} 2$ by the Oxford Clinical Cataract Classification and Grading System). ${ }^{8}$

Lamellar separation is best seen at the slit lamp with focal illumination (Fig. 1), when both the illuminating and viewing axes are somewhat oblique $\left(10^{\circ}\right.$ to $\left.20^{\circ}\right)$ to the optic axis of the eye. It presents the appearance of more or less parallel white lines. The lines measured from the macro-photographs and compensated for corneal magnification ${ }^{5}$ have a periodicity of $50-90 \mu \mathrm{m}$. The lines usually run approximately concentric with the lens equator. Thus they cross the direction of the lens fibres at a right angle. Some of the lines can be seen to branch and the branch lines sometimes rejoin their fellow line. The lines were most commonly found in the lower half of the lens.

Cortical spoke cataract and water clefts are commonly found in association with lamellar separation and the lines of lamellar separation are then seen to relate to these features. The lines commonly meet the radially running spoke or water cleft at close to a right angle and when they are at the same depth in the lens, the spoke or water cleft can be seen to interrupt the lines. The lines may cease at the spoke or water cleft, or they may continue on the other side with a small change in direction.

The lamellar separation lines also relate to the lens sutures in a similar way to their relationship with the spokes and water clefts. The lines either end at the suture or pass on with a small change in direction, which produces a 'herring bone' pattern (Fig. 1). Since the fibres of the lens join the suture lines obliquely, ${ }^{5,9}$ the 'herring bone' pattern shows that the lines are consistent in crossing the fibres at right angles on either side of the suture. In other places the lines peter out and no definite termination can be seen. When the lines are superficial to a spoke or water cleft, they can be seen to pass in front of the feature unaffected.

When the lines of lamellar separation are visualised at the microscope and the microscope then moved to a more oblique position, it is seen that the lines extend in depth into the lens. The lines of lamellar separation are not visible by retro-illumination and do not show at all with the retinoscope or with the slit lamp in retro-illumination mode.

The visualisation of the lines can be com- pletely abolished with polarising filters; showing that they are largely, or probably completely a reflective phenomenon. With colour film the lines of lamellar separation appear bluish, which shows that they are reflecting light preferentially towards the blue end of the spectrum.

\section{Associated Features (Table I)}

Lamellar separation was seen in all but one case to occur in association with the development of more obvious aspects of senile lens change, in particular cortical spoke cataract and/or water clefts. Lamellar separation was sometimes seen in lenses, which have very little cataract present. It may therefore be regarded as a feature of cortical cataract. Lamellar separation was not seen to be related to the occurrence of nuclear cataract.

It appears that lamellar separation does not have any significant effect on visual acuity on its own, but it usually occurs in conjunction with other pathological lens features that do affect visual acuity. The lack of visibility of the lines in retro-illumination at the slit lamp microscope also indicates that they do not interfere significantly with image formation.

To demonstrate that the observed lamellar separation lines were not an artefact due to handling, ${ }^{4}$ lenses without lamellar separation were deliberately mishandled and then re-examined. Lamellar separation was not observed in these lenses. Thus, it does not appear to be capable of being artificially induced by handling.

\section{Scanning Electron Microscopy Observations}

Of the nine lenses sent from Oxford with lamellar separation identified at the slit-lamp, lamellar separation was found in eight. In the masked control lens without lamellar separation, the condition was not found.

The visualisation of the ultrastructural changes in the lens fibres in lamellar separation largely depends on the fracture planes created during specimen preparation and therefore varies from lens to lens, The main ultrastructural features common to all lenses with lamellar separation are best appreciated in Figs. 2-5. A cross-fractured lens at low magnification (Fig. 2a) demonstrates that the 


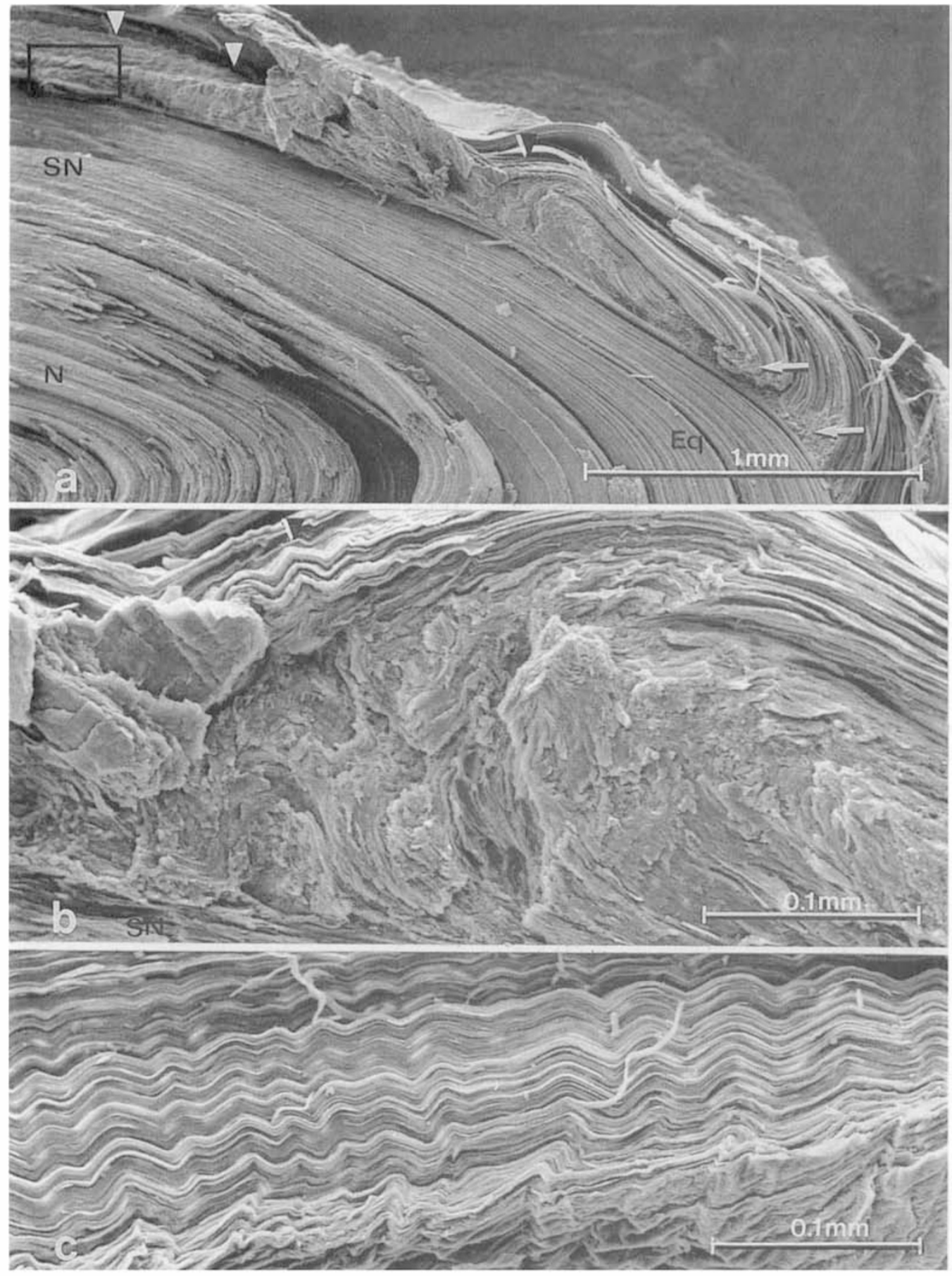

Fig. 2. (a) Low power view of a cross-fractured lens with lamellar separation. In the superficial equatorial region a large cohort of broken fibres is seen. Fibres with breaks, after an initial undisturbed course (arrows) become undulated anteriorly (arrowheads). The undisturbed parts of the broken fibres are superficial to an area of fully disturbed fibres (asterisks). ( $N=$ nuclear, $S N=$ supranuclear, $E q=$ equatorial).

(b) Medium power view of the region of disturbed fibres indicated in (a) (arrowheads).

(c) Medium power view of broken fibres in the anterior part of the lens (framed region in (a)). The folding of these fibres across their long axes is evident. 


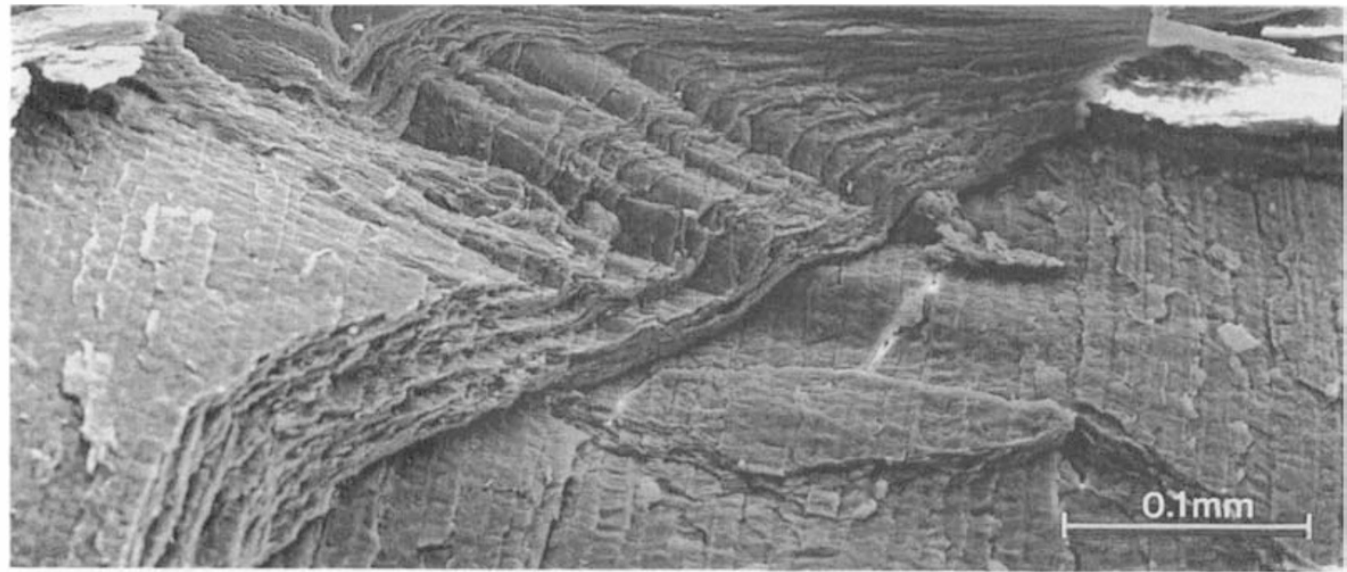

Fig. 3. Low power SEM view of folded fibres in the superficial anterior cortex of a human lens with lamellar separation. As compared to Fig. 5 fewer fibres are affected by folds and the folds extend less anteriorly.

fibres are broken in the equatorial region and are separated from their posterior parts. Deep to these fibres, the supranuclear and nuclear fibres are seen to be normal. The anterior course of the broken fibres is undisturbed for the first $1.0-2.0 \mathrm{~mm}$ and more anteriorly these fibres exhibit undulations or folds. At one point the folds are in close association with an area of undefined fibres. At higher magnifica- tion (Fig. 2b), this latter region shows a mass of fully disturbed fibres encompassed by the folded fibres and by the normal supranuclear fibres. The framed region of folded fibres in Fig. 2a is enlarged in Fig. 2c and clearly illustrates the undulation or folding of the fibres across their long axes giving rise to grooves and ridges in a large cohort of fibres. The number of fibres involved in these changes

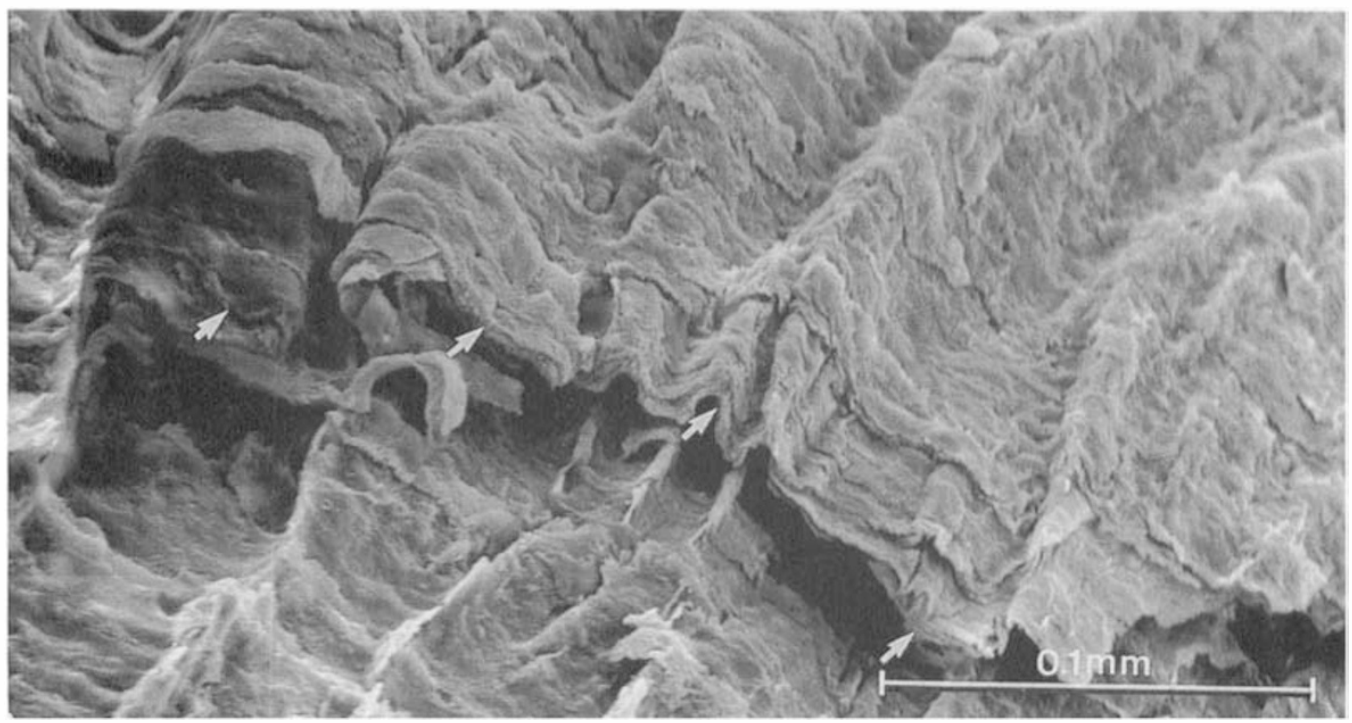

Fig. 4. Medium power SEM view of folded superficial anterior fibres in a lens with lamellar separation. Despite the extensive folding of the fibres, their cross-sectioned surface does not show the globular elements which characterise opaque fibres. 


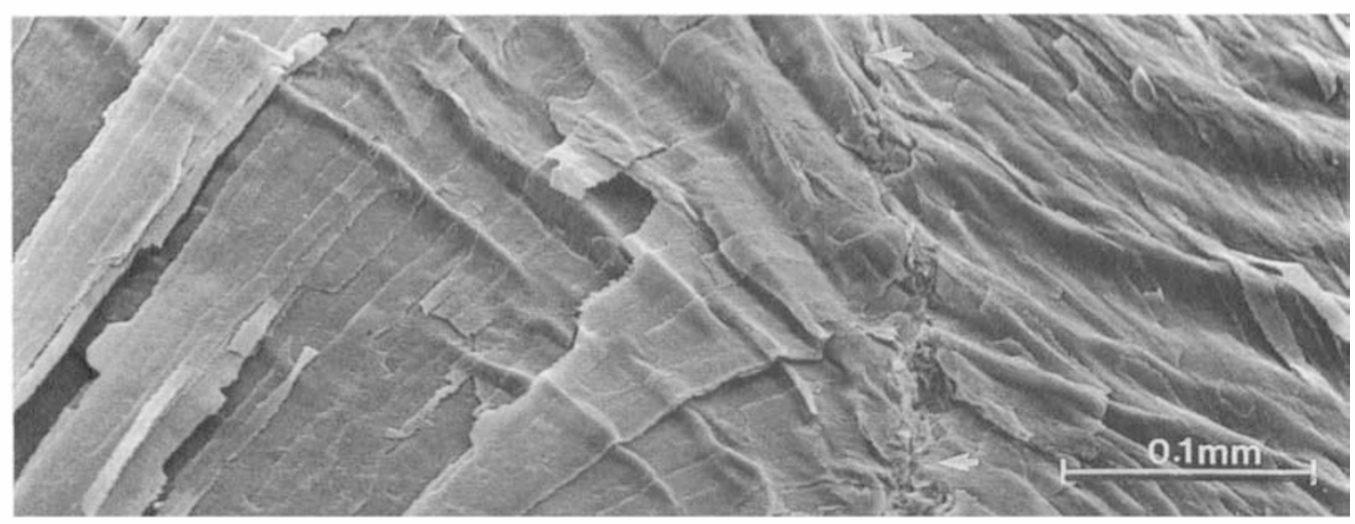

Fig. 5. Low power SEM view of human lens with lamellar separation fractured at a suture line, showing folding of the fibres on both sides of the suture. Note the smooth undisturbed ultrastructure of the folded fibres.

and their anterior extent varies from lens to lens.

The fibres adjacent to the broken and folded fibres are undisturbed and their membrane structure is unaffected (Figs. $3 \& 4$ ). The folded fibres (Fig. 4) are not filled by globular elements as is the case in spoke opacities. ${ }^{10}$

One lens (Fig. 5) was accidentally fractured at a suture line and shows fibre folding at different angles on either side of the slightly disturbed suture. This is in line with the 'herring bone' configuration seen at the slit-lamp (Fig. 1).

The angle between the incident electrons and the fibres is difficult to assess precisely and therefore the measurement of the distance between the ridges of the folds are rather approximate. Estimates of these distances from the pictures as in Fig. 2c gave values between $25-75 \mu \mathrm{m}$, which are of the same order of magnitude as those derived from the macrophotography $(50-90 \mu \mathrm{m})$.

The opacities, which are also present in the lenses have a similar SEM-ultrastructure to that described by many other authors in water clefts and in cohorts of affected fibres with large and small Morganian globules, as decribed in cataract by Harding et al. ${ }^{11}$ In addition we observed that the castor oil used for in vivo inspection was deleterious to the capsule, the epithelium and the first few fibre layers. As these changes were alike for control lenses and for lenses with lamellar separation, this effect by no means explained the ultrastructural changes reported here.

\section{Discussion}

The appearance of lamellar separation suggests that it is a reflective phenomenon that forms lines which cross the lens fibres at right angles and extends in depth into the lens as two dimensional plates. The visibility of the lines differs from that of cataract. When the polarisation filter technique is applied to cataract, as was shown by Weale, ${ }^{10}$ the visualisation of the cataract is reduced; showing that cataract is a partially reflective phenomenon. But the cataract does remain visible in polarised light because it scatters light which lamellar separation apparently does not.

It is difficult to imagine a lens fibre pathology that should affect the adjacent lens fibres, both on either side and in depth, in exactly the same way in each fibre. This could happen if the pathological defect in the lens fibres caused them to be banded and assembled together with the banding on each fibre level with that on the other fibres, but it does not seem likely that the lens fibre pathology would be so neatly arranged.

An insight into a possible explanation of the lamellar separation phenomenon came from considering the similarity between the lens cortex and the earth's crust, which is layed down in layers, or strata. In places the strata become folded, which ridges the surface. When the earth's surface ridging is looked on from above and the angle of lighting is low, 
the ridges are seen as more or less parallel lines, with occasional branching, an appearance similar to the lines of lamellar separation in the lens. Both the lines on the surface of the earth and those of the lens are continued in depth, which is a further aspect of their similarity. As the lens is transparent, the in depth continuation of the lines can be seen at the slit lamp. When the lens has been fractured for scanning electron microscopy, it is seen that the lines are due to folds in the lens fibres in similar way to the folds in the earth's crust. These folded lines in the lens fibres which are now clearly demonstrated by electron microscopy, have probably previously been seen by microscopists and electron microscopists, but dismissed as artefacts due to distortion of the material in the fixation process. In this study the folds were not found in the electron microscopy of the control lenses, which mitigates against their artifactual origin. The new name of 'Fibre Folds' is therefore proposed.

A small force would be needed to distort the lens fibres into folds and lines, but it is not obvious what this force may be. The force that is needed is one that tends to shorten each fibre so that it wrinkles. A possible distorting force may be provided by spoke cataracts or by water clefts. If these were areas of expansion they would transmit distorting forces into the lens fibres on either side. However, such a force would act at right angles to the direction of the lens fibres and would tend to compress, but not to shorten them.

It is known that lenses developing cataract are commonly smaller than healthy lenses of the same age $\mathrm{e}^{12,13,14}$ and this allows the possibility that shrinkage of lens substance is occurring, so that the lens fibres become too long for the new circumference on which they run and so wrinkle. Perkins ${ }^{14}$ observed that the lens size was not small in nuclear sclerotic cataract, which corresponds with the present observation that lamellar separation is not associated with nuclear sclerosis.

Gravity may play a part in the distribution of lamellar separation in the lens since in our study and in that of $\operatorname{Vogt}^{1}$ and Schild ${ }^{3}$ the lines are most commonly seen in the lower half of the lens. This would occur if slack fibres were sagging under gravity.

An alternative explanation to small lens size for the slackening of the lens fibres, is suggested in the present study by the presence of near equatorial breaks in the very lens fibres that are more centrally affected by folds. These breaks could release lens fibre tension and allow the lens fibres to sag and wrinkle. Preliminary observations in human donor lenses with early signs of opaque changes revealed that equatorial breaks are a common phenomenon in lenses exhibiting so called circular shades of Obazawa ${ }^{15}$ and that in most of these lenses fibre folds are present. In lenses exhibiting only early spoke opacities fibre folds are rare. The association of equatorial breaks and fibre folds may point to a causal relation between these phenomena.

The presence of the equatorial breaks may explain both the occurrence of fibre folds and of spoke catatarct which this study shows are strongly associated. Breakage of a lens fibre, or of a group of lens fibres, causes the opacity to run into those fibres from the break. ${ }^{16} \mathrm{~A}$ break in the periphery would be expected to produce a spoke shaped opacity. The relationship of spoke cataract to fibre breaks and to fibre folds will be the subject of a further study.

We would like to thank Professor David Easty and William Ayliffe, Bristol, for the supply of lenses.

\section{References}

${ }^{1}$ Vogt A: Lehrburch und Atlas der Spaltllampenmikroscopie des lebenden Auges. Vol 2. 1931 Springer Berlin, pp. 485-93.

${ }^{2}$ Berliner ML: Biomicroscopy of the eye. Vol 2. 1949, Hoeber, New York, pp. 1115-21.

${ }^{3}$ Schild H: Untersuchungen uber die Haufigkeit der lamellaren Zerkluftung, ihre Lage und Verlaufsrichtung in der vorderen und hinteren Linsenrinde. An 218 Augen sonst gesunder Personen. Arch f Ophthalmol 1921, 107: 49-50.

${ }^{4}$ Brown N: Macrophotography of the anterior segment of the eye. Br J Ophthalmol 1970, 54: 697701.

${ }^{5}$ Brown NAP, Bron AJ, Sparrow JM: An estimate of the size and shape of the human lens fibre in vivo. Br J Ophthalmol 1987, 71: 916-22.

${ }^{6}$ Willekens B, Vrensen G: The three dimensional organisation of lens fibres in the rhesus monkey. Graefe's Arch Clin Exp Ophthalmol 1982, 219: 112-20.

${ }^{7}$ Willekens B, Vrensen G: Lens fibre organisation in four avian species: a scanning electron microscopy study. Tissue Cell 1985, 17: 359-77.

${ }^{8}$ Sparrow JM, Bron AJB, Brown NAP, Ayliffe W, Hill AR: The Oxford clinical cataract classifica- 
tion and grading system. Int Ophthalmol 1986, 9: 207-25.

${ }^{9}$ Kuszak JR, Bertram BA, Mascai MS, Rae JL: Sutures of the crystalline lens: a review. Scanning Electron Microscopy 1984, 3: 1369-78.

${ }^{10}$ Weale RA: Real light scatter in the human crystalline lens. v. Graefe's Arch Clin Exptl Ophthalmol 1986, 224: 463-66.

${ }^{-11}$ Harding CV, Susan SR, Lo W-K, Bobrowski WF, Maisel H, Chylack LT Jr: The structure of the human cataractous lens. In the Ocular Lens, Structure, Function and Pathology. ed Harry Maisel. Ch.10. 1985 Marcel Dekker Inc. New York.

12 Weekers R, Delmarcelle J, Luyckx-Bacus J, Collignon J: Morphological changes of the lens with age and cataract. Ciba Foundation Symposium on The Human Lens in Relation to Cataract. 1973, 25-40.

${ }^{13}$ Brown N: Lens change with age and cataract. Ciba Foundation Symposium on The Human Lens in Relation to Cataract. 1973.65-71.

${ }^{14}$ Perkins ES: Lens thickness in early cataract. $\mathrm{Br} J$ Ophthalmol 1988, 72: 348-53.

${ }^{15}$ Obazawa H, Fujiwara T, Kawara T: The maturing process of the senile cataractous lens. Acta XXIV International Congress of Ophthalmology. Ed. Paul Henkind, American Academy of Ophthalmology. JB Lippincott 1983 Philadelphia.

${ }^{16}$ Bron AJ, Brown NAP: Lens structure and forms of cataract. EURAGE 1986. The Lens: Transparency and Cataract. Ed. G. Duncan. 1986. 3-11. 